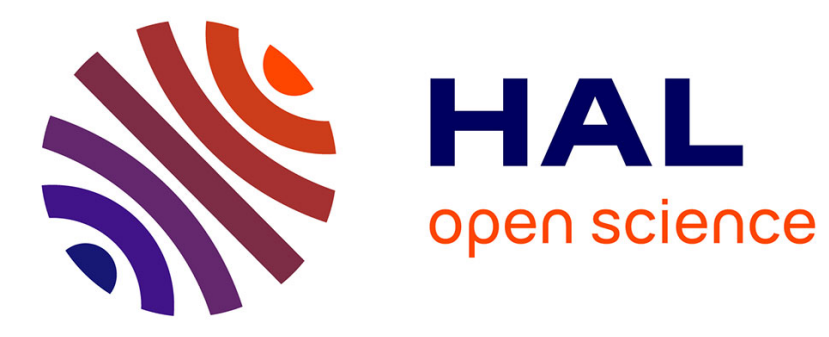

\title{
Qualité de vie et syndrome lipodystrophique chez les patients infectés par le VIH
}

\author{
Marie Préau, Anne Déborah Bouhnik, Bruno Spire, Catherine Leport, \\ Marianne Saves, Odile Picard, Jacques Reynes, Dominique Salmon, Pierre \\ Dellamonica, François Raffi, et al.
}

\section{To cite this version:}

Marie Préau, Anne Déborah Bouhnik, Bruno Spire, Catherine Leport, Marianne Saves, et al.. Qualité de vie et syndrome lipodystrophique chez les patients infectés par le VIH. L'Encéphale, 2006, 10.1016/S0013-7006(06)76223-6 . halshs-01563639

\section{HAL Id: halshs-01563639 \\ https://shs.hal.science/halshs-01563639}

Submitted on 17 Jul 2017

HAL is a multi-disciplinary open access archive for the deposit and dissemination of scientific research documents, whether they are published or not. The documents may come from teaching and research institutions in France or abroad, or from public or private research centers.
L'archive ouverte pluridisciplinaire HAL, est destinée au dépôt et à la diffusion de documents scientifiques de niveau recherche, publiés ou non, émanant des établissements d'enseignement et de recherche français ou étrangers, des laboratoires publics ou privés. 


\section{Qualité de vie et syndrome lipodystrophique chez les patients infectés par le VIH}

Article in L Encéphale · October 2006

DOI: 10.1016/S0013-7006(06)76223-6

CITATIONS

8

11 authors, including:

\section{Marie Préau}

Université Lumiere Lyon 2

155 PUBLICATIONS 1,121 CITATIONS

SEE PROFILE

\section{Patrizia Dellamonica}

Institute for Cancer Research and Treatment 162 PUBLICATIONS 1,016 CITATIONS

SEE PROFILE
READS

39

\section{Anne-Déborah Bouhnik}

French Institute of Health and Medical Resea...

127 PUBLICATIONS 1,153 CITATIONS

SEE PROFILE

François Raffi

513 PUBLICATIONS 9,743 CITATIONS

SEE PROFILE

Some of the authors of this publication are also working on these related projects: 
Qualité de vie et syndrome lipodystrophique chez les patients infectés par le VIH

L'Encéphale, 2006;32(5 Pt 1):713-9.

Marie PRÉAU ${ }^{1,2}$ Anne Déborah BOUHNIK ${ }^{2}$, Bruno SPIRE $^{2}$ Catherine LEPORT ${ }^{3}$, Marianne SAVES ${ }^{4}$, Odile PICARD ${ }^{5}$, Jacques REYNES ${ }^{6}$, Dominique SALMON ${ }^{7}$, Pierre DELLAMONICA $^{8}$, François RAFFI $^{9}$, Michel MORIN ${ }^{1,2}$ et le groupe d'étude APROCO-COPILOTE*

${ }^{1}$ Équipe Psychologie Sociale de la Santé, Laboratoire de Psychologie Sociale, Université de Provence, Aix en Provence

${ }^{2}$ INSERM U379/ORS PACA, Marseille

${ }^{3}$ Faculté X Bichat, Paris

${ }^{4}$ INSERM U593, Bordeaux

${ }^{5}$ Hôpital St Antoine, Paris

${ }^{6}$ Hôpital Gui de Chauliac, Montpellier

${ }^{7}$ Hôpital Cochin, Paris

${ }^{8}$ Hôpital Archet, Nice

${ }^{9}$ Hôtel Dieu, Nantes

$\underline{\text { Auteur correspondant : Marie PRÉAU }}$

INSERM U379/ORS PACA 23 rue Stanislas Torrents 13006 Marseille

Tel : 0682931405 / $0496102880 \quad$ Fax : 0496102899

@ preau@marseille.inserm.fr 


\section{Qualité de vie et syndrome lipodystrophique chez les patients infectés par le VIH}

\section{Résumé}

Ce travail vise à montrer ce qu'une évaluation psychosociale peut apporter à la compréhension de la subjectivité de la qualité de vie (QDV) des patients infectés par le VIH. Les analyses se sont appuyées sur les données de la cohorte APROCO-COPILOTE, incluant des patients infectés par le VIH initiant une multithérapie antirétrovirale. Ces nouvelles thérapies ont engendré, pour de nombreux patients, un type particulier d'effet indésirable : les lipodystrophies qui se caractérisent par des troubles de répartition de la masse graisseuse. L'objectif de ce travail est de déterminer les liens entre l'apparition de ce type d'effets indésirables et la QDV des personnes infectées par le VIH en distinguant selon qu'il s'agisse d'hommes ou de femmes. Les analyses effectuées sur un échantillon de 706 patients (ANCOVA) mettent en évidence un impact important mais différent des effets indésirables de type lipodystrophies sur la QDV et cela selon le type d'atteinte: lipodystrophie (accumulation) ou lipoatrophie (absence de graisse) et le sexe de la personne touchée. Il apparaît que, ajustée sur les caractéristiques socio-démographiques et psychosociales, la QDV des femmes est davantage affectée par les lipodystrophies alors que pour les hommes il s'agit avant tout des lipoatrophies. Ces résultats, interprétés au regard de la théorie corporo-sociopsychologique, montrent les implications déterminantes de ces effets indésirables quant à la QDV des patients infectés par le VIH. L'analyse des données empiriques a permis de mettre en évidence l'ancrage sociétal de l'évaluation de la QDV de par le rôle de l'entourage social, des soignants et du contexte social.

Mots clés : Qualité de vie, infection par le VIH, Effets secondaires, corporeité, théorie corporo-socio-psychologique 


\section{Health related quality of life and lipodystrophy syndrome among HIV-infected patients}

\section{Abstract}

The aim of this work is to show to what extent a psychosocial evaluation can bring to comprehension subjectivity of Quality of Life (QOL) among HIV-infected patients. Evaluation of QOL makes it possible to understand the link between the therapeutic effectiveness and the subjective evaluation of the treatment but also to estimate more precisely how people live and take their treatment in the context of HIV infection. This work confronts the variation of QOL with the variation of several social and psychosocial parameters identified as of the components of the system which is the subjective evaluation and more precisely to a specific side effect of highly active antiretroviral therapies (HAART): lipodystrophy syndrome that consists in body fat redistribution. This side effect could consist in an accumulation of body fat, or a loss of body fat or a combination of thsee two symptoms. The analysis was made on the data from APROCO-COPILOTE cohort composed of HIVinfected patients initiating HAART. Among a sample of 706 patients follow up for three years and with available QOL data, we identified the variations of QOL according to the variation of this specific side effect and according to gender. Results shows that lipodystrophy syndrome have a determinant impact on QOL different among male and female patients. Adjusted on clinical and socio-demographic characteristics, impaired women's QOL is associated with accumulation of body fat and impaired men's QOL is associated with loss of body fat. These results underline the role of body image on subjective evaluation of QOL. The analysis of empirical data made it possible to highlight the social implication of the evaluation of QOL from the role of the social support, patient-provider relationship and the social context.

Key words : Quality of life, HIV infection, self-reported side effects, body image 


\section{Qualité de vie et syndrome lipodystrophique chez les patients infectés par le VIH}

\section{Introduction}

Contraignant le patient à un traitement continu à long terme, l'utilisation des multithérapies antirétrovirales tend, à présent, à induire une évolution chronique de l'infection par le VIH qui est ainsi entrée dans une ère nouvelle souvent qualifiée de «normalisation » (Rosenbrock, Dubois-Arber et al. 2000; Setbon 2000). Cependant, le caractère chronique de la maladie et les contraintes du suivi et du traitement pèsent lourdement sur la vie quotidienne des personnes séropositives, frappées par la maladie souvent tôt dans l'âge adulte, brouillant les projets de vie et d'insertion sociale jusque dans leurs aspects les plus intimes. Les temps de la maladie sont des temps d'expérience subjective et la reconnaissance progressive de la charge psychosociale de cette dimension se traduit de plus en plus dans l'expansion de la notion de qualité de vie (QDV), compromis entre le point de vue des sciences sociales et celui de la médecine. La définition la plus connue attribuée à l'Organisation Mondiale de la Santé situe la QDV dans une perspective de santé publique et la définit comme «la perception qu'a l'individu de sa place dans l'existence, dans le contexte de la culture et du système de valeurs dans lesquels il vit, en relation avec ses objectifs, ses attentes, ses normes et ses inquiétudes » (World Health Organization 1984; Jasmin 1996). Dans cette définition très ouverte, la QDV est la perception de soi dans le monde, c'est une perception d'un soi social en terme de place. Cette perception est variable et déterminée par un ensemble complexe de facteurs qui caractérisent une position existentielle (Preau, Leport et al. 2004).

L'utilisation des multithérapies antirétrovirales (avec inhibiteurs de protéase ou analogues non nucléosidiques) engendre différents effets indésirables qui se différencient selon qu'ils apparaissent à court terme ou plutôt à long terme. Parmi les effets indésirables à long terme de 
ces traitements avec ou sans inhibiteur de protéase, on compte le syndrome lipodystrophique (Domingo, Perez et al. 1999; Thiebaut, Saves et al. 2003). Ce syndrome se caractérise par un trouble de la répartition des graisses souvent associé à des anomalies métaboliques. Les anomalies morphologiques rendent la maladie visible et se caractérisent par la perte du tissu adipeux sous-cutané, les lipo-atrophies et/ou l'accumulation de graisses au niveau de la ceinture abdominale par exemple, les lipohypertrophies (Collins, Wagner et al. 2000). Par ailleurs, ce syndrome est associé à des troubles biologiques métaboliques tels que l'hypercholestérolémie, l'hypertriglycéridémie ou encore l'intolérance aux hydrates de carbone (Saves, Raffi et al. 2002; Thiebaut, Saves et al. 2003). Sa prise en charge reste complexe et mal connue mais indispensable compte tenu des risques d'abandons thérapeutiques pour les patients qui accusent les multithérapies antirétrovirales de ces effets indésirables (Carr 2000; Duran, Spire et al. 2001; Blanch, Rousaud et al. 2002; Bodasing and Fox 2003; Carr 2003; Carr, Emery et al. 2003). Une modification du traitement est proposée lorsque cela est possible. Enfin, des techniques chirurgicales ou esthétiques pour les anomalies morphologiques sont proposées si la personne ne supporte plus ces transformations morphologiques sources d'une souffrance psychologique intense. Deux techniques réparatrices peuvent être proposées aux patients porteurs de lipoatrophies sous-cutanées notamment du visage : l'autogreffe de tissu adipeux ou Lipostructure ${ }^{\circledR}$ ou technique de Coleman d'une part, et l'injection de produit de comblement d'autre part.

Le syndrome lipodystrophique se manifestant soit par des lipoatrophies, soit par des lipohypertrophies, il est nécessaire dans les investigations sur la QDV de distinguer ces différents types d'anomalies morphologiques et d'en évaluer l'impact en terme de remise en cause de l'identité personnelle sur l'évaluation subjective de la QDV. Par ailleurs, on a pu mettre en évidence des différences de QDV selon le genre chez les patients infectés par le VIH (Shor-Posner, Lecusay et al. 2000; Cederfjall, Langius-Eklof et al. 2001; Cowdery and 
Pesa 2002; Preau, Leport et al. 2004) et plus particulièrement un effet du ressenti des effets indésirables spécifiquement auprès des femmes (Hudson, Kirksey et al. 2004). Il nous semble donc pertinent de tenter d'éclairer les raisons de ce décalage. Nous proposons l'hypothèse selon laquelle les éventuels ajustements et par conséquent les constructions de sens autour d'une nouvelle image corporelle engendrée par la survenue du syndrome lipodystrophie pourraient avoir un impact sur l'évaluation de la QDV.

L'objectif de cette étude consiste à déterminer les facteurs associés à une meilleure QDV chez les personnes infectées par le VIH durant la phase de maintenance de leur traitement en fonction de leur sexe, en explorant plus spécifiquement l'impact d'un effet indésirable particulier : le syndrome lipodystrophique.

\section{Matériel et méthodes}

Cette étude a consisté en une analyse transversale de données issues de la cohorte APROCO (AntiPROtéase Cohorte), cohorte d'observation prospective, multicentrique, nationale. L'objectif de cette cohorte est d'étudier l'évolution clinique et biologique, ainsi que les comportements d'observance des patients infectés par le VIH initiant un traitement par multithérapie avec inhibiteur de protéase (IP) dans le contexte de la pratique des prescriptions antirétrovirales en 1997. Les patients répondant aux critères d'inclusion dans les centres participants ont été inclus dans la cohorte. Les suivis médicaux (infection par le VIH, évènements cliniques et biologiques, prescriptions médicamenteuses) ont été effectués par recueil de données auprès de l'équipe médicale et un auto-questionnaire a été proposé aux patients. Ce dernier comprend des données socio-démographiques (âge, sexe, vie en couple, enfants), socio-économiques (emploi, logement), socio-comportementales et psychosociales. Ont été inclus, la version française de l'échelle de mesure de la QDV : SF-36 (Leplège, Ecosse et al. 1998), la présence de symptômes dépressifs (Échelle CES-D (Fuhrer and 
Rouillon 1989), des questions concernant le soutien social (famille et amis) ainsi que des questions concernant les relations avec le médecin et l'équipe médicale (Preau, Leport et al. 2004). L'échelle SF-36 est une échelle de QDV générique qui explore huit dimensions à partir desquelles il est possible de calculer deux scores synthétiques : un score agrégé de qualité de vie physique et un score agrégé de qualité de vie mentale. Il est aussi demandé au patient d'évaluer les effets indésirables du traitement à l'aide d'une échelle d'effets indésirables perçus construite spécifiquement par rapport à la problématique de l'infection à VIH dans le contexte des multithérapies. Cette échelle a été utilisée à de nombreuses reprises dans le cadre de la cohorte APROCO (Duran, Spire et al. 2001; Preau, Vincent et al. 2005).

\section{Analyses statistiques}

Une analyse des déterminants de la QDV trois ans après le début du traitement a été menée de manière transversale. Cette étude a été menée auprès des patients ayant répondu à l'auto-questionnaire posé après trois ans de traitement. Nous avons tout d'abord comparé les patients inclus dans notre analyse, c'est-à-dire les patients ayant rempli l'auto-questionnaire après trois ans de suivi - patients dits « répondants »- avec les patients de la cohorte exclus de notre analyse du fait de données manquantes à l'auto-questionnaire après trois ans de traitement - patients dits « non-répondants ».

Puis, afin de déterminer les facteurs indépendamment associés à une meilleure QDV mentale d'une part et à une meilleure QDV physique d'autre part, et en particulier de tester l'impact des effets indésirables de type lipodystrophies, des analyses de variance (ANOVA) et des analyses de covariance (ANCOVA) ont été menées conjointement chez les hommes et chez les femmes. L'ensemble des variables associées à la QDV (mentale ou physique) avec un p inférieur à 0,25 en analyse univariée étaient éligibles pour l'analyse multiple. 


\section{Résultats}

La population de cette recherche est composée de 706 patients de la cohorte APROCO. Sur les 1119 patients inclus dans la cohorte et ayant répondu à l'auto-questionnaire à l'inclusion, 706 ont répondu aux données de qualité de vie après trois ans sous traitement, ce qui représente $64 \%$ de l'échantillon. L'âge moyen de ces patients était de 38 ans, $79 \%$ des patients étaient des hommes, $58 \%$ bénéficiaient d'un emploi et $67 \%$ avaient un niveau d'études inférieur au baccalauréat. Les caractéristiques de ces patients sont disponibles dans le tableau 1.

Nous avons comparé les patients ayant répondu aux données de qualité de vie après trois ans de traitement aux patients qui n'ont pas répondu aux questions sur la qualité de vie, et cela afin de vérifier que ces patients dits « répondants » donc inclus dans cette étude n'étaient pas différents des patients «non-répondants », exclus de notre analyse.. Le tableau 1 montre que les patients répondants avaient plus fréquemment une situation professionnelle $(\mathrm{p}<0,001)$, qu'ils étaient davantage des hommes $(\mathrm{p}=0,038)$, plus fréquemment contaminés par relations homosexuelles $(\mathrm{p}=0,002)$. Ils étaient également plus âgés $(\mathrm{p}<0,001)$, avec un niveau d'études plus élevé ( $\mathrm{p}=0,004)$. D’un point de vue thérapeutique, ces patients étaient moins souvent des patients naïfs de traitement avant de commencer les multithérapies $(\mathrm{p}=0,002)$ ils présentaient de meilleures caractéristiques virologiques (charge virale plus basse) $(\mathrm{p}=0,045)$ et il s'agissait plus fréquemment de patients ayant atteint le stade SIDA $(p=0,029)$; en revanche, le taux de CD4 au début du traitement ne variait pas d'un groupe à l'autre $(\mathrm{p}=0,181)$.

\section{$\underline{\text { La qualité de vie mentale }}$}

Chez les hommes, le fait de rapporter un symptôme en lien avec la lipoatrophie, à savoir une atteinte objective de la corporéité en termes de perte de poids visible et subjective (notamment au niveau du visage) était indépendamment associé à une moins bonne QDV mentale après 
trois ans sous traitement (Tableau 2). Les autres facteurs indépendamment associés à une moins bonne QDV mentale étaient le fait de ne pas déclarer percevoir du soutien social de la part de sa famille et ses amis, ne pas être satisfait des explications de l'équipe médicale, ne pas avoir de logement confortable ainsi que le fait d'avoir été contaminé par usage de drogue. Chez les femmes, le fait d'avoir des lipohypertrophies, à savoir une atteinte de la corporeité était indépendamment associé à une moins bonne QDV mentale après trois ans sous traitement (Tableau 2). Le second facteur déterminant une bonne QDV mentale des femmes, en analyse multivariée, concernait le fait d'avoir une situation professionnelle.

\section{La qualité de vie physique}

Chez les hommes, le fait de rapporter au moins un symptôme en lien avec les lipoatrophies ou la lipohypertrophie, c'est à dire à la fois une accumulation de graisse sur certaines parties du corps mais aussi une absence totale de graisses sur d'autres parties du corps était indépendamment associé à une moins bonne QDV physique après trois ans sous traitement (Tableau 3). Les autres facteurs indépendamment associés à une moins bonne QDV physique consistaient dans le fait d'avoir des enfants, d'être contaminé depuis une longue période, de présenter des symptômes dépressifs ainsi que le fait d'avoir été contaminé par usage de drogue.

Chez les femmes, le fait de présenter un syndrome mixte à savoir lipodystrophies et lipoatrophique était indépendamment associé à une moins bonne QDV physique après trois ans sous traitement (Tableau 3). Les autres facteurs indépendamment associés à une moins bonne QDV physique étaient le fait de ne pas avoir un niveau d'étude supérieur au baccalauréat, ne pas avoir un logement confortable, présenter des caractéristiques immunologiques dégradées ainsi que des symptômes dépressifs. 


\section{Discussion}

Ces résultats mettent en évidence différents déterminants de la QDV mentale et physique des personnes infectées par le VIH et en phase de maintenance de leur traitement. Différents déterminants de la QDV, préalablement mis en évidence dans d'autres études ont été retrouvés dans cette recherche, tels que les relations médecin-patient ou le soutien social (Carrieri, Spire et al. 2003; Preau, Leport et al. 2004). Mais l'un des principaux facteurs, dont l'impact était peu connu jusqu'à présent, est le signalement d'un des effets indésirables le plus fréquent: le syndrome lipodystrophique. Ainsi, l'altération de l'image corporelle par la survenue d'effets indésirables des multithérapies antirétrovirales tels que les lipoatrophies ou les lipodystrophies a un effet particulièrement dommageable sur la QDV. Les résultats de cette étude montrent un effet particulièrement délétère des lipodystrophies sur la QDV physique et cela pour l'ensemble des patients, quel que soit leur sexe, les lipoatrophies ayant un effet significativement négatif sur la QDV physique pour les hommes. Chez les femmes, on peut présumer qu'il existe un effet plus fonctionnel des lipodystrophies qui affecte leur capacité physique et par là leur QDV physique.

Par ailleurs, la QDV mentale est principalement affectée par les lipoatrophies. Il semblerait que les hommes soient davantage affectés par les syndromes amaigrissants en comparaison avec les femmes. Ces résultats peuvent être interprétés en référence à la norme sociale de «profil corporel ». Les profils féminins plutôt minces sont socialement valorisés et les femmes souffrant de lipoatrophies sont moins nombreuses à être insatisfaites ou gênées par cette perte de poids, même si elle n'affecte que certaines parties du corps.

L'infection par le VIH compromet l'estime de soi au sens où elle remet en question l'intégrité structurale du corps ainsi que ses capacités d'attraction physique et sexuelle. Ainsi, dans le processus d'attrait exercé par la beauté, la présence et la perception du visage jouent un rôle privilégié. Sur un plan très général Simmel a cherché à dégager «la signification esthétique 
du visage » (Simmel 1988). «Dans le corps humain, le visage est ce qui possède au plus haut point une unité intrinsèque » (Simmel 1988). La preuve en est qu'une modification ou une déformation même minime peut changer radicalement son caractère et son expression. En outre « grâce à sa mobilité et à son pouvoir d'expression, il est seul à devenir comme le lieu géométrique de la personnalité intime » (Maisonneuve et Bruchon-Schweitzer 1999). Ainsi, le visage constitue sans doute la part la plus singulière de l'apparence, il permet d'identifier chacun même dans un champ social étendu et en deçà du langage.

Si l'on se réfère au modèle corporo-psycho-social proposé par Santiago (2002), la remise en cause de la corporéité atteint à la fois l'identité personnelle et sociale. Pour les patients infectés par le VIH, il est fondamental de rappeler que la remise en cause de l'identité sociale passe par le souci des autres qui peuvent toujours devenir des juges stigmatisants (Oette, Juretzko et al. 2002). Ainsi, comme le souligne Santiago : «corporéité, émotion et rapport à autrui semblent liés dès la naissance (...) l'ancrage de l'être humain dans sa corporéité et sa sociabilité soutient un modèle de l'être humain incarné, conçu comme un système ouvert sur la relation au monde et sur son milieu et ses transformations» (Santiago-Delefosse 2000) p.275. La modélisation de l'ensemble de ces paramètres a permis à Santiago de proposer le modèle «corporo-socio-psychologique» au sein duquel est intégrée la dimension d'un « milieu bio » en tant qu'élément d'un système social qui en fait un milieu «corporo-sociopsychologique ». Comme le précise cette dernière, la spécificité de ce modèle réside en le fait qu'il intègre l'énergétique, le dévelopemental et le vécu concret humain; et cela tout en modélisant les liens entre corporéité, affectivité/cognition et socialisation. En effet, les ajustements au monde comme les différentes manières de faire face à un évènement difficile dépendent non seulement du sujet mais également des interprétations subjectives de sa corporéité et des intégrations possibles ou non des systèmes cognitifs et émotiono-affectifs 
(Santiago-Delefosse 2000). Tel que le développe ce modèle et tout comme le décrit l'approche phénoménologique appliquée au malade, les modifications corporelles engendrées par l'expérience de la maladie et des traitements nécessitent pour le sujet une réadaptation de l'identité et notamment dans son rapport à l'environnement social.

Par ailleurs, de par leurs effets indirects, et au delà de la problématique de la QDV, ces changements quant à l'image corporelle peuvent avoir un impact sur la volonté des patients de poursuivre leur traitement, comme cela a pu être mis en évidence précédemment (Martinez, Garcia-Viejo et al. 2001) ou sur l'observance au traitement (Duran, Spire et al. 2001).

Il existe des limites méthodologiques à cette étude.

Tout d'abord, l'instrument utilisé, l'échelle SF-36, est une échelle de mesure générique de la QDV, qui ne permet pas de tenir compte des problématiques spécifiques de l'infection par le VIH, telles que les problèmes liés à la sexualité et qui paraissent particulièrement importants dans le cadre d'une population plutôt jeune.

Par ailleurs, l'échantillon de patients auprès duquel a été effectué cette étude, peut ne pas être tout à fait représentatif de la population française infectée par le VIH. En effet, les patients inclus dans cette cohorte devaient initier un traitement avec inhibiteur de protéase, actuellement, de nombreux patients ne sont plus sous inhibiteur de protéase, les molécules disponibles sont plus nombreuses et ne présentent pas exactement les mêmes effets indésirables, malgré cela le syndrome lipodystrophique demeure un problème tout à fait actuel pour ces patients.

Ces résultats mettent en évidence le rôle déterminant de certains types d'effets indésirables des multithérapies antirétrovirales qui peuvent altérer l'image de soi des personnes concernées 
et par là même affecter leur QDV. Comme cela a pu être montré dans d'autres études auprès de la même cohorte, la QDV reste déterminée par différents facteurs psychosociaux, tels que la relation avec le médecin, ainsi que le contexte social et la précarité sociale dans lesquels vivent certains patients (Carrieri, Spire et al. 2003; Preau, Leport et al. 2004). 


\section{Références bibliographiques}

Blanch, J., A. Rousaud, et al. (2002). "Impact of lipodystrophy on the quality of life of HIV-1-infected patients." Journal of Acquired Immune Deficiency Syndrome 31(4): 404-7.

Bodasing, N. and R. Fox (2003). "HIV-associated lipodystrophy syndrome: assessment and management." Journal of Infection 46(2): 87-93.

Carr, A. (2000). "HIV protease inhibitor-related lipodystrophy syndrome." Clinical Infectious Disease 30 Suppl 2: S135-42.

Carr, A. (2003). "HIV lipodystrophy: risk factors, pathogenesis, diagnosis and management." Aids 17 Suppl 1 : S141-8.

Carr, A., S. Emery, et al. (2003). "An objective case definition of lipodystrophy in HIV-infected adults: a casecontrol study." Lancet 361(9359): 726-35.

Carrieri, P., B. Spire, et al. (2003). "Health-related quality of life after 1 year of highly active antiretroviral therapy." Journal of Acquired Immune Deficiency Syndrome 32(1): 38-47.

Cederfjall, C., A. Langius-Eklof, et al. (2001). "Gender differences in perceived health-related quality of life among patients with HIV infection." AIDS Patient Care STDS 15(1): 31-9.

Collins, E., C. Wagner, et al. (2000). "Psychosocial impact of the lipodystrophy syndrome in HIV infection." AIDS Read 10(9): 546-50.

Cowdery, J. E. and J. A. Pesa (2002). "Assessing quality of life in women living with HIV infection." $\underline{\text { AIDS }}$ Care 14(2): 235-45.

Domingo, P., A. Perez, et al. (1999). "Lipodystrophy in HIV-1-infected patients." Lancet 354(9181): 868.

Duran, S., B. Spire, et al. (2001). "Self-reported symptoms after initiation of a protease inhibitor in HIV-infected patients and their impact on adherence to HAART." HIV Clinical Trials 2(1): 38-45.

Fuhrer, R. and F. Rouillon (1989). "La version française de l'échelle CES-D. Description et traduction de l' echelle d' autoevaluation." Psychiatrie et Psychobiologie 4: 163-166.

Hudson, A., K. Kirksey, et al. (2004). "The influence of symptoms on quality of life among HIV-infected women." West J Nurs Res 26(1): 9-23; discussion 24-30.

Jasmin, C. (1996). Cancer et sida : des modèles pour l'étude de la qualité de vie. Cancer, Sida. La qualité de vie. C. Jasmin, Jay, A., Bez, G. Paris, Synthélabo Groupe, Les empecheurs de penser en rond: 17-22.

Leplège, A., E. Ecosse, et al. (1998). "The French SF-36 Health Survey: translation, cultural adaptation and preliminary psychometric evaluation." Journal of Clinical Epidemiology 51(11): 1013-23.

Maisonneuve, J. and M. Bruchon-Schweitzer (1999). Le corps et la beauté. Paris, PUF.

Martinez, E., M. A. Garcia-Viejo, et al. (2001). "Lipodystrophy syndrome in patients with HIV infection: quality of life issues." Drug Safety 24(3): 157-66.

Oette, M., P. Juretzko, et al. (2002). "Lipodystrophy syndrome and self-assessment of well-being and physical appearance in HIV-positive patients." AIDS Patient Care STDS 16(9): 413-7.

Preau, M., C. Leport, et al. (2004). "Health-related quality of life and patient-provider relationships in HIVinfected patients during the first three years after starting PI-containing antiretroviral treatment." $\underline{\text { AIDS }}$ Care 16(5): 649-61.

Preau, M., E. Vincent, et al. (2005). "Health-related quality of life and health locus of control beliefs among HIV-infected treated patients." J Psychosom Res 59(6): 407-13.

Rosenbrock, R., F. Dubois-Arber, et al. (2000). "The normalization of AIDS in Western European countries." Social Science and Medicine 50(11): 1607-29.

Santiago-Delefosse, M. (2000). "Actualité d' un modele wallonien des emotions : Vers un modele corporopsycho-social des emotions." L'Encephale 26(1): 8-20.

Saves, M., F. Raffi, et al. (2002). "Factors related to lipodystrophy and metabolic alterations in patients with human immunodeficiency virus infection receiving highly active antiretroviral therapy." Clin Infect Dis 34(10): 1396-405.

Setbon, M. (2000). "La normalisation paradoxale du sida." Revue Française de Sociologie 41(1): 61-78.

Shor-Posner, G., R. Lecusay, et al. (2000). "Quality of life measures in the Miami HIV-1 infected drug abusers cohort: relationship to gender and disease status." Journal of Substance Abuse 11(4): 395-404.

Simmel, G. (1988). La tragédie de la culture. Paris, Editions Rivages.

Thiebaut, R., M. Saves, et al. (2003). "Epidémiologie du risque vasculaire d'origine atherosclereuse chez les patients infectés par le VIH-1." La Presse Medicale 32(30): 1419-26.

World Health Organization (1984). Report of the Working Group on Concepts and Principles of Health Promotion. Copenhagen, WHO. 
Tableau 1. Comparaison des caractéristiques des patients répondants et non-répondants dans l'étude sur la qualité de vie selon le sexe après trois ans sous multithérapies antirétrovirale (Cohorte APROCO, n=1119)

\begin{tabular}{|c|c|c|c|}
\hline & $\frac{\begin{array}{c}\text { Patients } \\
\text { Non-répondants }\end{array}}{(1)}$ & $\frac{\frac{\text { Patients }}{\text { Répondants }}}{(2)}$ & (1) vs (2) \\
\hline & 413 & 706 & \\
\hline Age moy $(\sigma)$ & $\begin{array}{c}37,2(8,3) \\
\mathrm{n}(\%)\end{array}$ & $\begin{array}{c}38,4(9,2) \\
\mathrm{n}(\%)\end{array}$ & $<0,001^{a}$ \\
\hline Hommes (vs femmes) & $306(74)$ & $561(79)$ & $\mathbf{0 , 0 3 8}{ }^{\mathrm{b}}$ \\
\hline Emploi (vs non) & $220(53)$ & $410(58)$ & $<0,001^{\mathrm{b}}$ \\
\hline $\begin{array}{l}\text { Charge virale (log copies/ml), } \\
\text { med[IQR] }\end{array}$ & $4,6[3,8 ; 5,2]$ & $4,4[3,6 ; 5,1]$ & $\mathbf{0 , 0 4 5} 5^{\mathrm{a}}$ \\
\hline Niveau d'études < bac ( vs $\geq$ bac) & $309(75)$ & $469(67)$ & $0,004^{b}$ \\
\hline $\mathbf{C D} 4 / \mathbf{m m}^{3}<200(\mathrm{vs} \geq 200)$ & $153(37)$ & $234(33)$ & $0,181^{b}$ \\
\hline \multicolumn{4}{|l|}{ Groupe de transmission } \\
\hline Utilisateurs de drogue & $91(22)$ & $107(15)$ & $\mathbf{0 , 0 0 2}{ }^{\mathrm{b}}$ \\
\hline Homosexuels & $146(35)$ & $309(44)$ & \\
\hline Hétérosexuels & $143(35)$ & $216(31)$ & \\
\hline Autres & $33(8)$ & $74(10)$ & \\
\hline $\begin{array}{l}\text { Naiff de traitement antiretroviral (vs } \\
\text { non) }\end{array}$ & $203(49)$ & $280(40)$ & $\mathbf{0 , 0 0 2}{ }^{b}$ \\
\hline Stade clinique C (vs A ou B) & $79(19)$ & $141(20)$ & $\mathbf{0 , 0 2 9 ^ { b }}$ \\
\hline
\end{tabular}


Tableau 2. Facteurs indépendamment associés à une meilleure QDV mentale après 3 ans sous multithérapies antirétrovirale (Cohorte APROCO ; n=706, ANCOVA multiple)

QDV mentale à Mo

Emploi à M0

Logement confortable à $\mathrm{M} 0$

Groupe de transmission

Utilisateur de drogue IV

Homosexuels

Hétérosexuels

Autres

Satisfaction des explications de l'équipe médicale

Important soutien social du partenaire, de la famille et des amis

Lipodystrophies
Femmes

$\mathrm{n}=145(\mathbf{2 0 , 5} \%)$

$\mathbf{R}^{2}=\mathbf{0 , 4 1}$

\begin{tabular}{cccc}
\hline $\boldsymbol{\beta}[\mathrm{IC} 95 \%]$ & $\mathbf{p}$ & $\boldsymbol{\beta}[\mathrm{IC} 95 \%]$ & $\mathbf{p}$ \\
\hline $0,33[0,25 ; 0,42]$ & $<0,001$ & $0,63[0,47 ; 0,79]$ & $<0,001$ \\
& & $3,3[-0,2 ; 6,8]$ & 0,064
\end{tabular}

$2,1[0,2 ; 4,1] \quad 0,033$

1

$3,4[0,4 ; 6,3] \quad 0,024$

$3,5[0,1 ; 6,9] \quad 0,045$

$5,0[1,0 ; 8,9] \quad 0,014$

$2,7[0,8 ; 4,7] \quad 0,005$

$3,9[1,9 ; 5,9] \quad<0,001$

$-4,2[-6,1 ;-2,3]<0,001$ 
Tableau 3. Facteurs indépendamment associés à une meilleure QDV physique après 3 ans sous multithérapies antirétrovirale (Cohorte APROCO ; n=706, ANCOVA multiple)

\begin{tabular}{|c|c|c|c|c|}
\hline & \multicolumn{2}{|c|}{$\begin{array}{c}\text { Hommes } \\
\mathbf{n = 5 6 1}(\mathbf{7 9 , 5 \% )} \\
\mathbf{R}^{2}=\mathbf{0 , 3 4}\end{array}$} & \multicolumn{2}{|c|}{$\begin{array}{c}\text { Femmes } \\
\mathrm{n}=145(20,5 \%) \\
\mathbf{R}^{2}=\mathbf{0 , 4 6}\end{array}$} \\
\hline & $\boldsymbol{\beta}[\mathrm{IC} 95 \%]$ & $\mathbf{p}$ & $\boldsymbol{\beta}[\mathrm{IC} 95 \%]$ & $\mathbf{p}$ \\
\hline QDV physique à M0 & $0,30[0,23 ; 0,37]$ & $<0,001$ & $0,43[0,28 ; 0,59]$ & $<0,001$ \\
\hline Enfant à M0 & $-2,1[-3,5 ;-0,6]$ & 0,005 & & \\
\hline Niveau d'étude > bac & & & $4,0[1,9 ; 6,9]$ & 0,007 \\
\hline Groupe de transmission & & & & ns \\
\hline Utilisateur de drogue IV & 1 & & & \\
\hline Homosexuels & $3,0[1,0 ; 5,0]$ & 0,004 & & \\
\hline Hétérosexuels & $1,9[-0,3 ; 4,2]$ & 0,096 & & \\
\hline Autres & $1,6[-1,0 ; 4,2]$ & 0,220 & & \\
\hline $\begin{array}{l}\text { Temps depuis le diagnostic } \\
\text { de séropositivité } \geq 8 \text { ans }\end{array}$ & $-1,8[-3,3 ;-0,3]$ & 0,018 & & $\mathrm{~ns}$ \\
\hline $\mathrm{CD} 4+\geq 200 \mathrm{~mm} 3$ & & & $6,9[0,5 ; 13,3]$ & 0,035 \\
\hline $\begin{array}{l}\text { Symptomatologie } \\
\text { dépressive }\end{array}$ & $-1,5[-2,8 ;-0,2]$ & 0,028 & $-3,3[-6,0 ;-0,6]$ & 0,017 \\
\hline $\begin{array}{l}\text { Syndrome } \\
\text { lipodystrophique }\end{array}$ & & & $-3,6[-6,5 ;-0,7]$ & 0,015 \\
\hline Lipoatrophies & $-2,0[-3,4 ;-0,6]$ & 0,004 & & ns \\
\hline Lipodystrophies & $-1,6[-3,0 ;-0,2]$ & 0,022 & $-3,3[-6,4 ;-0,3]$ & 0,031 \\
\hline
\end{tabular}

